\section{Sportler und Präventionsexperte werben für mehr Bewegung Mit dem Schrittzähler gegen ein erhöhtes Diabetesrisiko}

- Eigenlob, Ansporn durch das Umfeld und gegebenenfalls ein Schrittzähler: Wer sein Diabetesrisiko senken möchte, muss alles tun, um am Ball zu bleiben. „Theoretisch wissen wir alle, was wir unseren Patienten empfehlen sollten, wenn ein erhöhtes Diabetesrisiko festgestellt wird“, sagte Prof. Peter Schwarz, Dresden. Mit 150 Minuten Sport pro Woche, verteilt auf sechs Einheiten à 30 Minuten, und dazu einer Gewichtsreduktion um 5-7\% könne der Typ-2-Diabetes oft verhindert werden.

Das Problem ist freilich die Umsetzung. Vor allem Menschen, die viele Jahre lang körperlich nicht aktiv waren, tun sich schwer, ein konsequentes Training zu starten. Der ehemalige Olympia-Zweite im Zehnkampf und heutiger Sportlicher Leiter des Deutschen Zentrums für Präventivmedizin in Damp an der Ostsee, Frank Busemann, gab in Berlin Tipps, wie ein Sportprogramm aussehen sollte, das Aussicht auf Erfolg hat: „Man sollt nichts überstürzen, vor allem wenn man vorher jahrzehntelang keinen Sport gemacht hat." Wichtig ist auch, die Motivation nicht durch unrealistische Erwartungen zu gefährden: „Wer seine Ziele zu hoch steckt, verliert schnell den Spaß am neuen Hobby." Statt übertriebenen Ehrgeiz ist Eigenlob angesagt: „Man sollte sich ruhig selbst auf die Schulter klopfen, wenn man sein Pensum absolviert hat. Das hilft."

Schließlich sollte auch der normale Alltag stärker genutzt werden, um sich zu bewegen. Kleine Sportübungen am Arbeitsplatz, Treppen statt Aufzug und Verzicht auf unnötige Verkehrsmittel lauten hier die Empfehlungen. Gerade im Alltag leistet auch ein Schrittzähler gute Dienste. Schwarz zeigte seinen eigenen Schrittzähler vor: „Seit ich den am Gürtel habe, mache ich tausend Schritte mehr am Tag als vorher." Optimal seien an einem normalen Arbeitstag etwa 10000 Schritte.

Effektive Prävention des Diabetes ist freilich nur möglich, wenn Menschen mit erhöhtem Risiko auch rechtzeitig erkannt werden. Die von Sanofi-Aventis initiierte Kampagne „Wissen was bei Diabetes zählt: Gesünder unter 7“ hat sich dem verschrieben - auch bei der diesjährigen Leichtathletik-WM 2009 als nationaler Partner.

gvg

Veranstaltung „Vorbeugung, Früherkennung und Leben mit Diabetes“, 2009 in Berlin, Veranstalter: Sanofi-Aventis

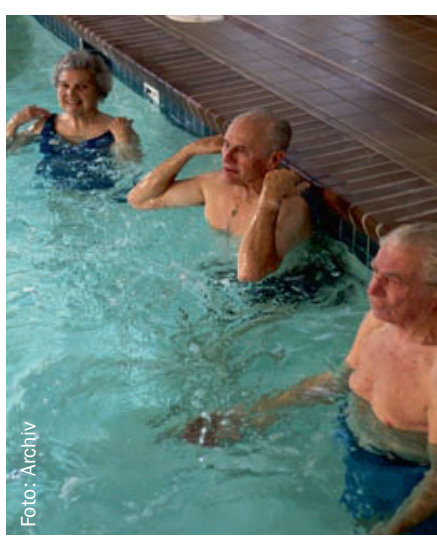

Wer lange keinen Sport gemacht hat, sollte vorsichtig damit anfangen.

\title{
Hypoglykämie \\ Unterschätzte Gefahr bei Diabetes mellitus Typ 2
}

- Das Risiko von Hypoglykämien wird bei Patienten mit Diabetes Typ 2 unterschätzt, v.a. bei der Therapie mit Sulfonylharnstoffen und Insulin. Schwere Hypoglykämien sind jedoch mit einer erhöhten kardiovaskulären Letalität assoziiert.

Hypoglykämien sind für den Patienten extrem unangenehm, betonte Prof. Martin Pfohl, Duisburg. Als ,leicht“ wird eine Hypoglykämie dann bezeichnet, wenn noch eine Selbsttherapie durch den $\mathrm{Pa}$ tienten möglich ist. Die schwere Form ist durch zentralnervöse Symptome und Bewusstlosigkeit gekennzeichnet. Nach der Definition der DDG liegt eine Hypoglykämie dann vor, wenn der Blutzuckerspiegel $<50 \mathrm{mg} / \mathrm{dl}(<2,8 \mathrm{mmol} / \mathrm{l})$ sinkt. Dies ist jedoch viel zu niedrig, so Pfohl. Viele Patienten könnten bei einem solch niedrigen Blutzuckerspiegel gar nicht mehr reagieren. Pfohl rät daher, sich an den Definitionen der American Diabetes Association (ADA) zu orientieren. Dort liegt eine Hypoglykämie bei $<70 \mathrm{mg} / \mathrm{dl}(<3,9 \mathrm{mmol} / \mathrm{l})$ vor.

Eine Hypoglykämie gefährdet Patienten nicht nur wegen eines erhöhten Unfallrisikos, sondern auch durch klinische Folgen. So hatten Patienten in der ACCORD-Studie mit schweren Hypoglyk- ämien unabhängig vom Behandlungsarm eine höhere Sterblichkeit (ohne Hypoglykämien im Intensivarm 1,3\%, mit 2,8\%, im Standardarm 1,1 bzw. $4,9 \%)$. Die genauen Ursachen hierfür sind noch unklar. Mehrere Untersuchungen an Patienten mit Herzinfarkten belegen jedoch, dass Hypoglykämien in der Akutphase ebenfalls mit einer erhöhten Letalität und Reinfarktrate einhergehen.

„In Deutschland leisten wir uns den Luxus, dass wir - abgesehen von Metformin - vor allem auf zwei Substanzen setzen, die ein erhöhtes Hypoglykämierisiko haben: Glibenclamid und Insulin“ so Pfohl. Bei den etwa 5\% Patienten mit Diabetes Typ 2, die durch Hypoglykämien gefährdet sind, sollte man nicht dabei bleiben. Für eine normnahe Einstellung $(80-110 \mathrm{mg} / \mathrm{dl})$ sollten in Kombination mit Metformin bevorzugt Substanzen mit niedrigem Hypoglykämierisiko eingesetzt werden, wie Rosiglitazon (Avandia ${ }^{\circledR}$ ). Studien zeigen, dass sich so das Hypoglykämierisiko um bis zu $80 \%$ senken lässt. suhl

Pressegespräch „Diabetesbehandlung: Wo stehen wir heute, wohin geht die Therapie von morgen?“, 2009 in München, Veranstalter: GlaxoSmithKline 